\title{
NEURO-IMAGES
}

\section{How Does the Brain Die After a Massive Posterior Fossa Lesion?}

\author{
Chris Marcellino ${ }^{1,2} \mathbb{D}$, Sherri A. Braksick ${ }^{1}$ and Eelco F. M. Wijdicks ${ }^{1 *}$ \\ (c) 2020 Springer Science+Business Media, LLC, part of Springer Nature and Neurocritical Care Society
}

A devastating neurological complication occurred [1-4] as a result of the hypercoagulability of COVID-19 pneumonia [5]. An ischemic stroke (or perhaps venous sinus thrombosis) involved the posterior inferior cerebellar artery (PICA) territory with hemorrhagic conversion, severe mass effect, and subsequent brain death despite maximal medical therapy. Rarely $[6,7]$ seen sequential imaging shows the radiographic development of supratentorial destruction from a posterior fossa hemorrhagic infarction.

\section{Description of the Case}

A 61-year-old woman with a pertinent history of metabolic syndrome was hospitalized due to severe hypoxemia from COVID-19 pneumonia, diagnosed by positive nasal swab for SARS-CoV-2 and typical ground glass opacities on chest computed tomography (CT) imaging. She was transferred to the ICU on the 3rd day of hospitalization and was found to have a D-Dimer serum level above the upper limit of detection $(>42,000 \mathrm{ng} / \mathrm{mL}$ Fibrinogen Equivalent Units) despite having received subcutaneous prophylactic dose heparin since the time of admission. Imaging confirmed the absence of deep venous thrombosis and pulmonary emboli. She was intubated on the 5th hospital day after failing noninvasive oxygen therapy. Despite deep sedation and paralysis, she required prone positioning for refractory hypoxemia by the 6th day of hospitalization. Management of her respiratory and renal failure necessitated nearly continuous deep sedation, neuromuscular blockade, and renal replacement therapy. On the evening of the 11th day of hospitalization, sedation was weaned at which point she

\footnotetext{
*Correspondence: wijde@mayo.edu

${ }^{1}$ Division of Neurocritical Care and Hospital Neurology, Department of Neurology, Mayo Clinic, 200 First Street SW, Rochester, MN 55905, USA Full list of author information is available at the end of the article
}

resumed some spontaneous movements as she had during prior sedation holidays. However, early in the morning of the 12th day, movement ceased and she became unresponsive.

CT of the head was performed, which showed a large right cerebellar intraparenchymal hemorrhage with extensive mass effect resulting in basilar cistern effacement and upward transtentorial herniation (Fig. 1a). There was marked hydrocephalus and early loss of posterior fossa gray-white differentiation (Fig. 1b-d). The edema surrounding the hemorrhage was confined to the PICA territory, raising suspicion that a PICA or venous sinus thrombosis underwent hemorrhagic conversion. On exam, only cough and gag reflexes as well as the right corneal reflex were preserved, without central or peripheral motor response to pain. A ventriculostomy was placed as the patient was not a surgical candidate (due to neurological condition, coagulopathy, and multiorgan failure). There was brisk drainage of the ventriculostomy and intracranial pressures (ICPs) measured approximately $30 \mathrm{mmHg}$ despite medical therapy. The neurologic examination progressed to loss of all brainstem reflexes by the 13th day, which was followed by ICP measurements less than $10 \mathrm{mmHg}$. On the 17th day, CT angiography was obtained which showed diffuse cerebral edema and near complete ventricular collapse, but filling only to the M1 segments bilaterally (Fig. 2a-e).

Despite maximal preoxygenation, she rapidly desaturated in less than a minute with a closed circuit held on end inspiration with maintained expiratory pressure, suggesting that even with tracheal insufflation, an apnea test could not be tolerated in fulminant COVID-19 pneumonia and acute respiratory distress syndrome (ARDS) without use of mechanical ventilation. The remainder of the brainstem reflex and motor exam was completed with only negative findings, and confounding variables were systematically ruled out [8]. With a confirmatory nuclear

\section{Springer}



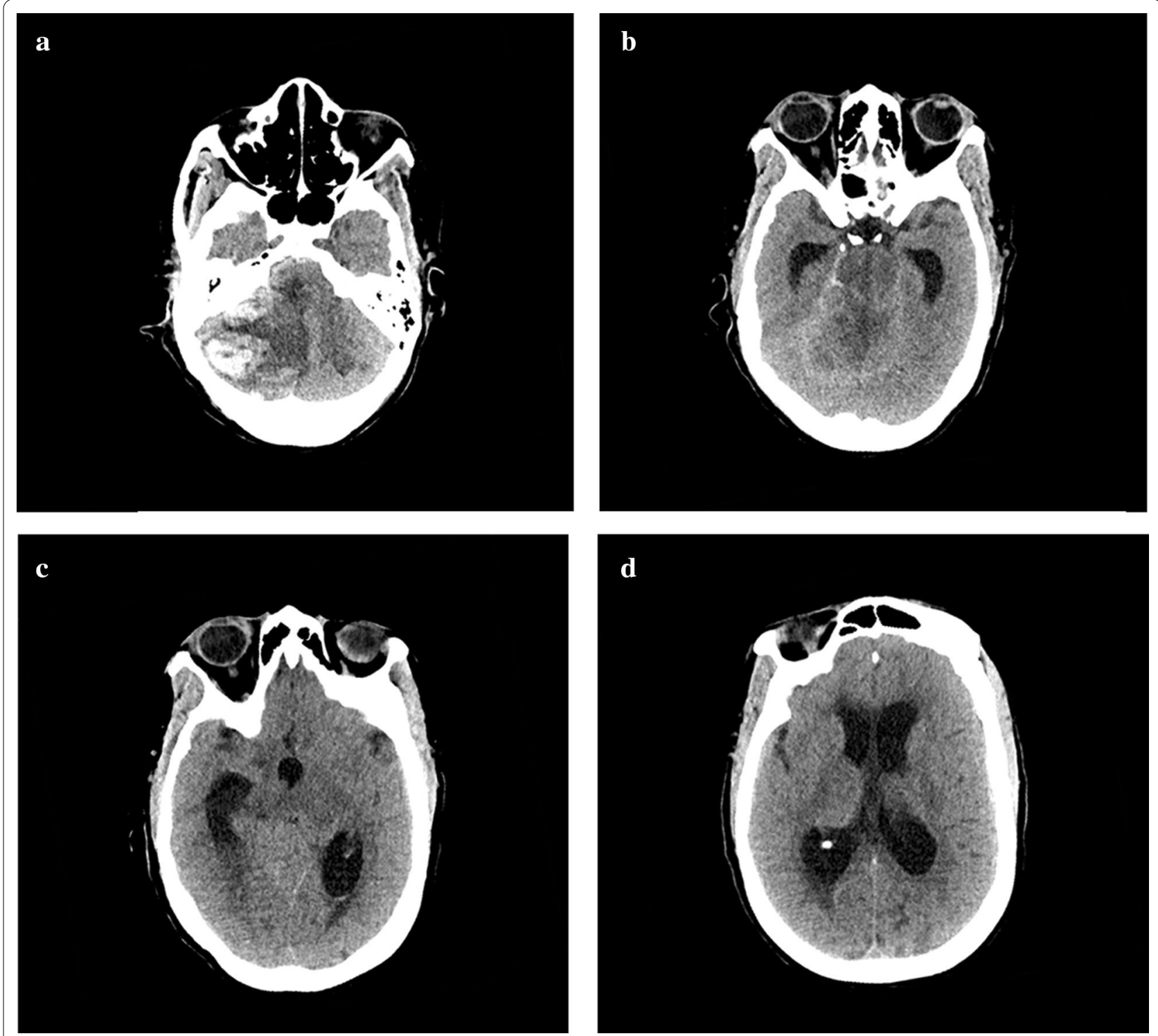

Fig. 1 Axial CT head images from the 12th day of hospitalization a probable established right PICA territory infarction or posterior fossa venous sinus thrombosis with hemorrhagic conversion (measuring $3.5 \times 2.9 \mathrm{~cm}$ on coronal images) resulting in basilar cistern effacement and upward transtentorial herniation. b-d Early loss of gray-white differentiation isolated to the posterior fossa and tri-ventricular hydrocephalus is seen in higher slices. This scan was performed prior to ventriculostomy

study showing no uptake, brain death was declared on the 19th day of hospitalization (Fig. 3a, b).

\section{Discussion}

Our case provided the opportunity to observe a sequence of events after presumed primary brainstem death, which is seldom captured sequentially with modern CT imaging. Neuroimaging showed the progression of brain destruction caused by an expansive posterior fossa mass causing upward (through the tentorial opening) and downward herniation (through the foramen magnum). Despite maximal cerebrospinal fluid (CSF) drainage, diffuse cerebral edema obliterated the ventricular system with initially elevated ICP and later low ICP after herniation. The process of hemispheric infarction from an acute posterior fossa mass is illustrated by the sequence of radiographic images: 

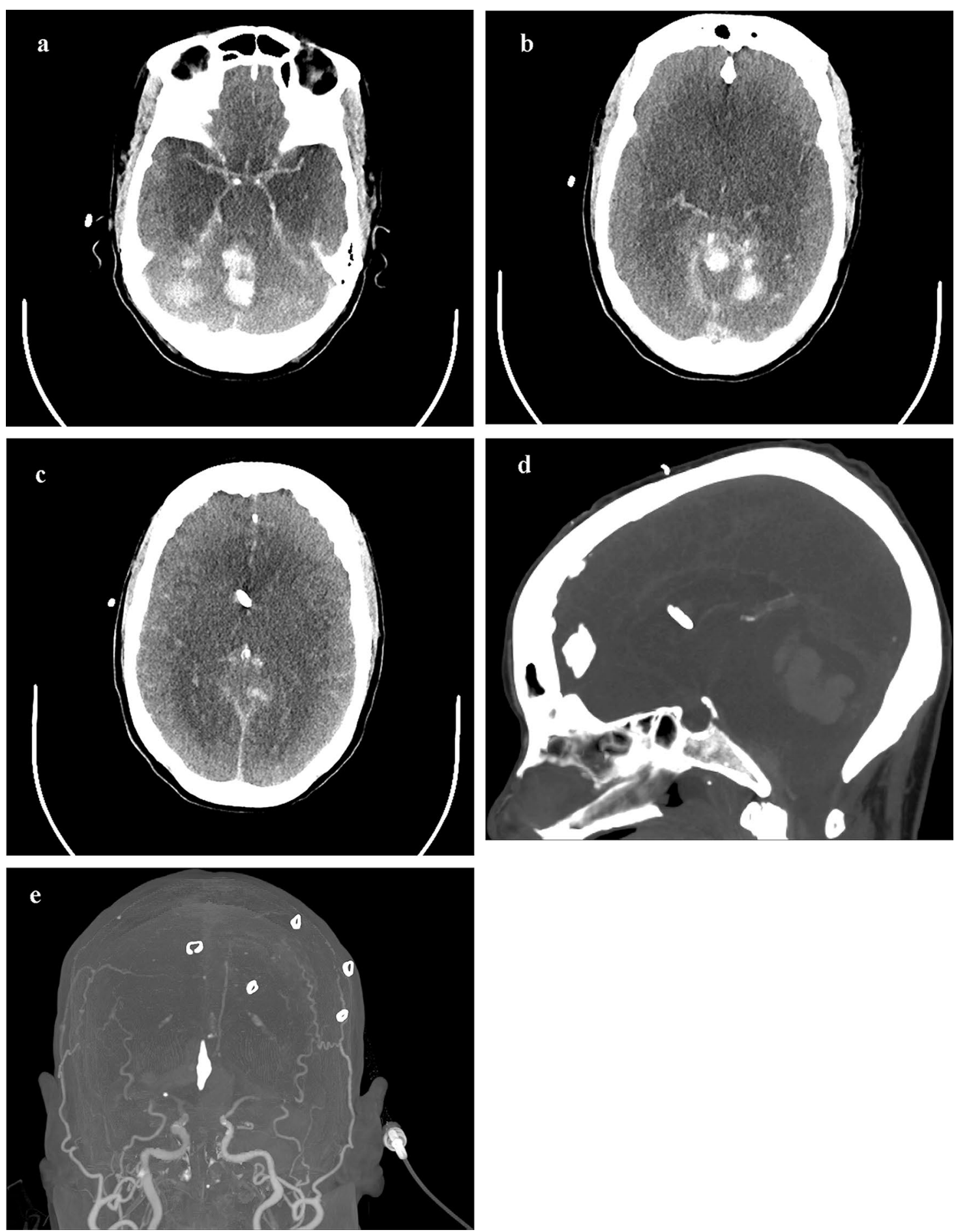

Fig. 2 a-c Axial, d reconstructed sagittal and $\mathbf{e}$ coronal CT angiography head images from the 17th day of hospitalization, after the interval loss of all brainstem reflexes which showed diffuse cerebral edema and near complete ventricular collapse, but preserved filling of the Circle of Willis and M1 segments without further evidence of cortical perfusion, which are also demonstrated in $\mathbf{f}$ A-P 3-D reconstructions. The sagittal images demonstrate extensive upward herniation and compression on the deep venous structures 


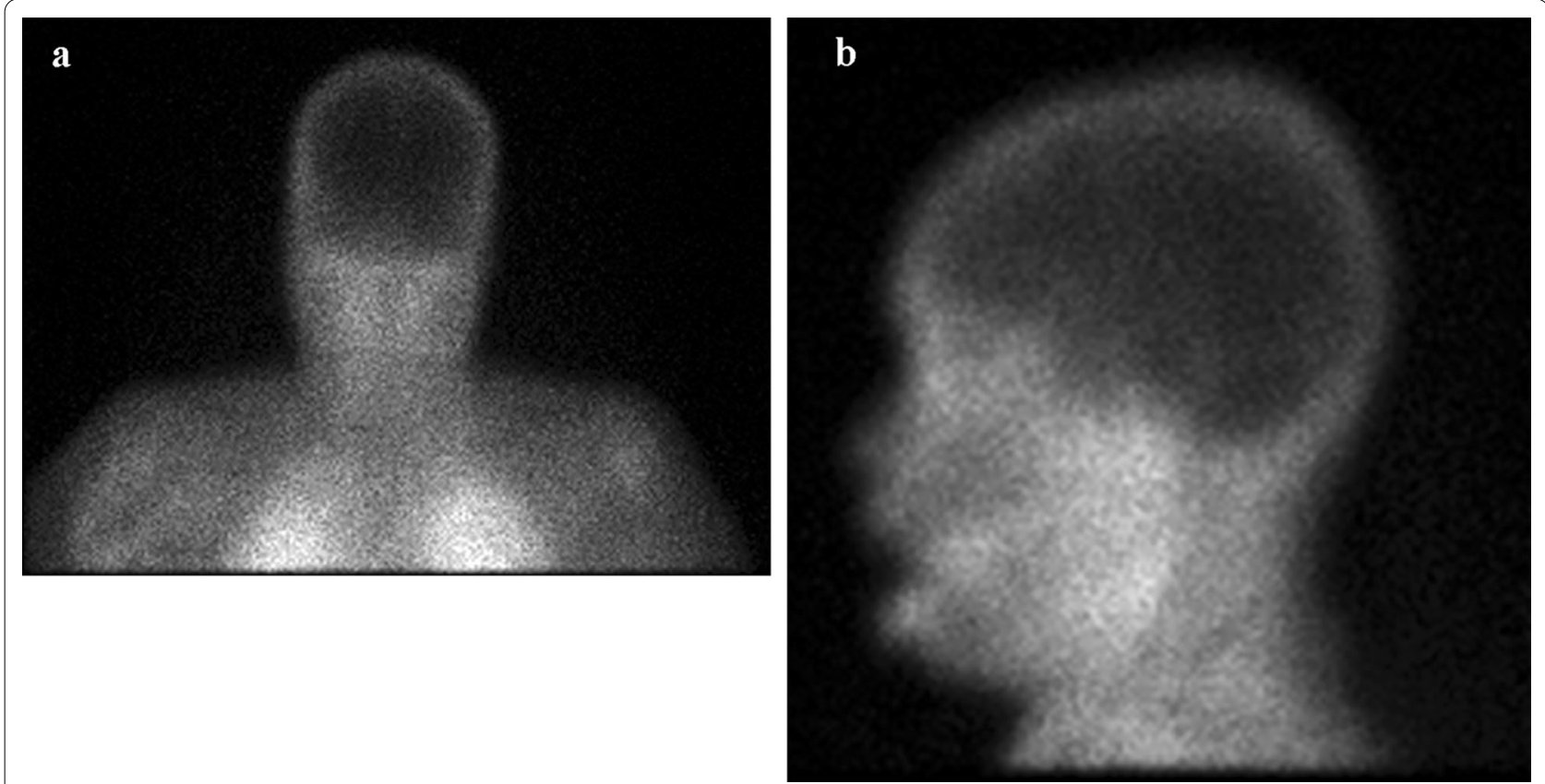

Fig. 3 Technetium 99M radionuclide study $\mathbf{a}$ coronal and $\mathbf{b}$ lateral demonstrating the absence of cerebral blood flow

1. Multiple cerebellar and brainstem infarcts and mass effect from hemorrhagic conversion of the right cerebellum rapidly produces progressive and extensive loss of brainstem function (Fig. 1a, b).

2. Secondary hydrocephalus from tentorial compression and aqueductal obstruction from upward herniation, and by implication compression of the vein of Galen, causes venous congestion and thus a multifactorial increase in ICP, which was not improved with ventriculostomy (Fig. $1 \mathrm{~b}-\mathrm{d}$ ).

3. Increased ICP impedes intracranial blood flow into the anterior circulation beyond the M1 segments (Fig. 2a-f). The rest of the brain is seriously irrevocably under-perfused. The posterior circulation was already non-filled at this time.

4. Total brain infarction ensues and blood flow stops secondary to diffuse cerebral edema, proven via radionuclide study (Fig. 3a, b).

Most patients who are declared brain dead have a supratentorial mass lesion or diffuse process that results in infratentorial destruction, but our patient showed the opposite. These images depict the full process of brain infarction from a posterior fossa etiology, and this process is more multifaceted than acute hydrocephalus alone. Once compression nearly totally destroyed the brainstem, the swelling rapidly and indirectly destroys the rest of the brain via upward herniation, compression of the vein of Galen and venous congestion. In such a multifaceted brain injury, ventriculostomy will not improve the condition nor will decompression of the cerebellum. A better understanding of the pathophysiology of posterior fossa lesions with loss of all brainstem reflexes is important in evaluating brain death $[7,9]$. This case again confirms that when an examination shows complete loss of brainstem reflexes in a massive posterior fossa lesion, a conserved and likely very predictable progression occurs with no posterior, followed by no anterior, cerebral perfusion. In primary brainstem death (from intrinsic lesions or compression), the revelant vital functions have ceased and other functions are on the verge of ceasing.

Fulminant COVID-19 pneumonia poses logistical challenges in the brain death examination due to unsafe apnea testing in patients with dramatic and diffuse exudative epithelial denudation of alveoli. The COVID-19 pandemic has dramatically reduced organ donation [10], but the effect on the frequency of brain death determination is not known in both COVID-19 positive and negative patients.

\section{Author details}

${ }^{1}$ Division of Neurocritical Care and Hospital Neurology, Department of Neurology, Mayo Clinic, 200 First Street SW, Rochester, MN 55905, USA. ${ }^{2}$ Department of Neurologic Surgery, Mayo Clinic, 200 First Street SW, Rochester, MN 55902, USA. 


\section{Author Contributions}

$\mathrm{CM}$ drafted the manuscript. All authors revised the manuscript, and participated in the medical care of the patient.

\section{Source of support}

No funding was required for the study.

\section{Compliance with Ethical Standards}

\section{Conflict of interest}

The authors declare that there is no conflict of interest.

\section{Informed consent}

Not applicable; no identifying information is included in this article.

\section{Publisher's Note}

Springer Nature remains neutral with regard to jurisdictional claims in published maps and institutional affiliations.

Received: 13 July 2020 Accepted: 30 October 2020

Published online: 2 December 2020

\section{References}

1. Benger M, Williams $O$, Siddiqui J, Sztriha L. Intracerebral haemorrhage (ICH) and COVID-19: clinical characteristics from a case series. Brain Behav Immunity. 2020;88:940-4.
2. Oxley TJ, Mocco J, Majidi S, et al. Large-vessel stroke as a presenting feature of Covid-19 in the young. New Engl J Med. 2020;382:e60.

3. Mao L, Jin H, Wang M, et al. Neurologic manifestations of hospitalized patients with coronavirus disease 2019 in Wuhan, China. JAMA Neurol. 2020;77:683-90.

4. Lodigiani C, lapichino G, Carenzo L, et al. Venous and arterial thromboembolic complications in COVID-19 patients admitted to an academic hospital in Milan, Italy. Thromb Res. 2020;191:9-14.

5. Wang C, Horby PW, Hayden FG, Gao GF. A novel coronavirus outbreak of global health concern. Lancet. 2020;395:470-3.

6. Manara A, Varelas P, Wijdicks EF. Brain death in patients with "isolated" brainstem lesions: a case against controversy. J Neurosurg Anesthesiol. 2019:31:171-3.

7. Varelas PN, Brady P, Rehman M, et al. Primary posterior fossa lesions and preserved supratentorial cerebral blood flow: implications for brain death determination. Neurocrit Care. 2017;27:407-14.

8. Wijdicks EF, Varelas PN, Gronseth GS, Greer DM. Evidence-based guideline update: determining brain death in adults - report of the Quality Standards Subcommittee of the American Academy of Neurology. Neurology. 2010:74:1911-8.

9. Walter U, Fernández-Torre JL, Kirschstein T, Laureys S. When is "brainstem death" brain death? The case for ancillary testing in primary infratentorial brain lesion. Clin Neurophysiol. 2018;129:2451-65.

10. Loupy A, Aubert O, Reese PP, Bastien O, Bayer F, Jacquelinet C. Organ procurement and transplantation during the COVID-19 pandemic. Lancet. 2020;395:e95-6. 